\title{
Research Article \\ Solitons, Peakons, and Periodic Cuspons of a Generalized Degasperis-Procesi Equation
}

\author{
Jiangbo Zhou and Lixin Tian \\ Nonlinear Scientific Research Center, Faculty of Science, Jiangsu University, Zhenjiang, \\ Jiangsu 212013, China \\ Correspondence should be addressed to Jiangbo Zhou, zhoujiangbo@yahoo.cn
}

Received 24 November 2008; Accepted 23 February 2009

Recommended by Elbert E. Neher Macau

We employ the bifurcation theory of planar dynamical systems to investigate the exact travelling wave solutions of a generalized Degasperis-Procesi equation $u_{t}-u_{x x t}+4 u u_{x}+\gamma\left(u-u_{x x}\right)_{x}=3 u_{x} u_{x x}+$ $u u_{x x x}$. The implicit expression of smooth soliton solutions is given. The explicit expressions of peaked soliton solutions and periodic cuspon solutions are also obtained. Further, we show the relationship among the smooth soliton solutions, the peaked soliton solutions, and the periodic cuspon solutions. The physical relevance of the found solutions and the reason why these solutions can exist in this equation are also given.

Copyright (C 2009 J. Zhou and L. Tian. This is an open access article distributed under the Creative Commons Attribution License, which permits unrestricted use, distribution, and reproduction in any medium, provided the original work is properly cited.

\section{Introduction}

Recently, Degasperis and Procesi [1] derived a nonlinear dispersive equation

$$
u_{t}-u_{x x t}+4 u u_{x}=3 u_{x} u_{x x}+u u_{x x x}
$$

which is called the Degasperis-Procesi equation. Here $u(t, x)$ represents the fluid velocity at time $t$ in the $x$ direction in appropriate nondimensional units (or, equivalently the height of the water's free surface above a flat bottom). The nonlinear convection term $u u_{x}$ in (1.1) causes the steepening of wave form, whereas the nonlinear dispersion effect term $3 u_{x} u_{x x}+u u_{x x x}=\left((1 / 2) u^{2}\right)_{x x x}$ in (1.1) makes the wave form spread. Equation (1.1) can be regarded as a model for nonlinear shallow water dynamics. Degasperis et al. [2] showed that the (1.1) is integrable by deriving a Lax pair and a bi-Hamiltonian structure for it. Yin proved local well posedness to (1.1) with initial data $u_{0} \in H^{s}(\mathbb{R}), s>3$ on the line [3] and on the circle [4]. The global existence of strong solutions and weak solutions to (1.1) is investigated in [4-10]. The solution to Cauchy problem of (1.1) can also blow up in finite time when the initial data satisfies certain sign condition[7-10]. Vakhnenko and Parkes [11] obtained 
periodic and solitary-wave solutions of (1.1). Matsuno [12,13] obtained multisoliton, cusp and loop soliton solutions of (1.1). Lundmark and Szmigielski [14] investigated multipeakon solutions of (1.1). Lenells [15] classified all weak travelling wave solutions. The shock wave solutions of (1.1) are investigated in [16,17].

Yu and Tian [18] investigated the following generalized Degasperis-Procesi equation:

$$
u_{t}-u_{x x t}+4 u u_{x}=3 u_{x} u_{x x}+u u_{x x x}-\gamma u_{x x x}
$$

where $r$ is a real constant, and the term $u_{x x x}$ denotes the linear dispersive effect. They obtained peaked soliton solutions and period cuspon solutions of (1.2). Unfortunately, they did not obtain smooth soliton solutions of (1.2).

In this paper, we are interesting in the following generalized Degasperis-Procesi equation:

$$
u_{t}-u_{x x t}+4 u u_{x}+\gamma\left(u-u_{x x}\right)_{x}=3 u_{x} u_{x x}+u u_{x x x}
$$

where $\gamma$ is a real constant, the term $u_{x}$ denotes the dissipative effect and the term $u_{x x x}$ represents the linear dispersive effect. Employing the bifurcation theory of planar dynamical systems, we obtain the analytic expressions of smooth solitons, peaked solitons, and period cuspons of (1.3). Our work covers and supplements the results obtained in [18].

The remainder of the paper is organized as follows. In Section 2, using the travelling wave transformation, we transform (1.3) into the planar dynamical system (2.3) and then discuss bifurcations of phase portraits of system (2.3). In Section 3, we obtain the implicit expression of smooth solitons, the explicit expressions of peaked solitons and periodic cuspon solutions. At the same time, we show that the limits of smooth solitons and periodic cusp waves are peaked solitons. In Section 4, we discuss the physical relevance of the found solutions and give the reason why these solutions can exist in (1.3).

\section{Bifurcations of Phase Portraits of System (2.3)}

We look for travelling wave solutions of (1.3) in the form of $u(x, t)=\varphi(x-c t)=\varphi(\xi)$, where $c$ is the wave speed and $\xi=x-c t$. Substituting $u=\varphi(\xi)$ into (1.3), we obtain

$$
-c \varphi^{\prime}+c \varphi^{\prime \prime \prime}+4 \varphi \varphi^{\prime}-3 \varphi^{\prime} \varphi^{\prime \prime}-\varphi \varphi^{\prime \prime \prime}+\gamma \varphi^{\prime}-\gamma \varphi^{\prime \prime \prime}=0
$$

By integrating (2.1) once we have

$$
\varphi^{\prime \prime}(\varphi-c+\gamma)=g-(c-\gamma) \varphi+2 \varphi^{2}-\left(\varphi^{\prime}\right)^{2}
$$

where $g$ is the integral constant. 
Let $y=\varphi^{\prime}$, then we get the following planar dynamical system:

$$
\begin{aligned}
& \frac{d \varphi}{d \xi}=y, \\
& \frac{d y}{d \xi}=\frac{g-(c-\gamma) \varphi+2 \varphi^{2}-y^{2}}{\varphi-c+\gamma},
\end{aligned}
$$

with a first integral

$$
H(\varphi, y)=(\varphi-c+\gamma)^{2}\left(y^{2}-\varphi^{2}-g\right)=h,
$$

where $h$ is a constant.

Note that (2.3) has a singular line $\varphi=c-\gamma$. To avoid the line temporarily we make transformation $d \xi=(\varphi-c+\gamma) d \zeta$. Under this transformation, (2.3) becomes

$$
\begin{aligned}
& \frac{d \varphi}{d \zeta}=(\varphi-c+\gamma) y, \\
& \frac{d y}{d \zeta}=g-(c-\gamma) \varphi+2 \varphi^{2}-y^{2} .
\end{aligned}
$$

System (2.3) and system (2.5) have the same first integral as (2.4). Consequently, system (2.5) has the same topological phase portraits as system (2.3) except for the straight line $\varphi=c-\gamma$. Obviously, $\varphi=c-\gamma$ is an invariant straight-line solution for system (2.5).

For a fixed $h,(2.4)$ determines a set of invariant curves of system (2.5). As $h$ is varied, (2.4) determines different families of orbits of system (2.5) having different dynamical behaviors. Let $M\left(\varphi_{e}, y_{e}\right)$ be the coefficient matrix of the linearized system of (2.5) at the equilibrium point $\left(\varphi_{e}, y_{e}\right)$, then

$$
M\left(\varphi_{e}, y_{e}\right)=\left(\begin{array}{cc}
y_{e} & \varphi_{e}-c+\gamma \\
4 \varphi_{e}-c+\gamma & -2 y_{e}
\end{array}\right)
$$

and at this equilibrium point, we have

$$
\begin{aligned}
& J\left(\varphi_{e}, y_{e}\right)=\operatorname{det} M\left(\varphi_{e}, y_{e}\right)=-2 y_{e}^{2}-\left(\varphi_{e}-c+\gamma\right)\left(4 \varphi_{e}-c+\gamma\right), \\
& p\left(\varphi_{e}, y_{e}\right)=\operatorname{trace}\left(M\left(\varphi_{e}, y_{e}\right)\right)=-y_{e} .
\end{aligned}
$$

By the qualitative theory of differential equations (see [19]), for an equilibrium point of a planar dynamical system, if $J<0$, then this equilibrium point is a saddle point; it is a center point if $J>0$ and $p=0$; if $J=0$ and the Poincare index of the equilibrium point is 0 , then it is a cusp. 
By using the first integral value and properties of equilibrium points, we obtain the bifurcation curves as follows:

$$
\begin{aligned}
& g_{1}(c)=\frac{(c-\gamma)^{2}}{8}, \\
& g_{2}(c)=-(c-\gamma)^{2} .
\end{aligned}
$$

Obviously, the two curves have no intersection point and $g_{2}(c)<0<g_{1}(c)$ for arbitrary constants $c \neq \gamma$.

Using bifurcation method of vector fields (e.g., [19]), we have the following result which describes the locations and properties of the singular points of system (2.5).

Theorem 2.1. For a given constant wave speed $c \neq 0$, let

$$
\begin{gathered}
\varphi_{0}^{ \pm}=\frac{c-\gamma \pm \sqrt{(c-\gamma)^{2}-8 g}}{4} \quad \text { for } g \leq g_{1}(c), \\
y_{0}^{ \pm}= \pm \sqrt{(c-\gamma)^{2}+g} \quad \text { for } g \geq g_{2}(c) .
\end{gathered}
$$

When $c=\gamma$,

(1) if $g<0$, then system (2.5) has two equilibrium points $(-\sqrt{-g / 2}, 0)$ and $(\sqrt{-g / 2}, 0)$, which are saddle points;

(2) if $g=0$, then system (2.5) has only one equilibrium point $(0,0)$, which is a cusp;

(3) if $g>0$, then system (2.5) has two equilibrium points $(0,-\sqrt{g})$ and $(0, \sqrt{g})$, which are saddle points.

When $c \neq \gamma$,

(1) if $g<g_{2}(c)$, then system (2.5) has two equilibrium points $\left(\varphi_{0}^{-}, 0\right)$ and $\left(\varphi_{0}^{+}, 0\right)$. They are saddle points:

(i) if $c>\gamma$, then $\varphi_{0}^{-}<-1 / 2(c-\gamma)<1 / 4(c-\gamma)<c-\gamma<\varphi_{0}^{+}$,

(ii) if $c<\gamma$, then $\varphi_{0}^{-}<c-\gamma<1 / 4(c-\gamma)<-1 / 2(c-\gamma)<\varphi_{0}^{+}$;

(2) if $g=g_{2}(c)$, then system (2.5) has three equilibrium points $\left(\varphi_{0}^{-}, 0\right),\left(\varphi_{0}^{+}, 0\right)$, and $(c-\gamma, 0)$. $(c-r, 0)$ is a cusp:

(i) if $c>\gamma$, then $\varphi_{0}^{-}=-(1 / 2)(c-\gamma)<(1 / 4)(c-\gamma)<c-\gamma=\varphi_{0}^{+}$. $\left(\varphi_{0}^{-}, 0\right)$ is a saddle point, while $\left(\varphi_{0}^{+}, 0\right)$ is a degenerate center point,

(ii) if $c<\gamma$, then $\varphi_{0}^{-}=c-\gamma<1 / 4(c-\gamma)<-1 / 2(c-\gamma)=\varphi_{0}^{+}$. $\left(\varphi_{0}^{-}, 0\right)$ is a degenerate center point, while $\left(\varphi_{0}^{+}, 0\right)$ is a saddle point; 


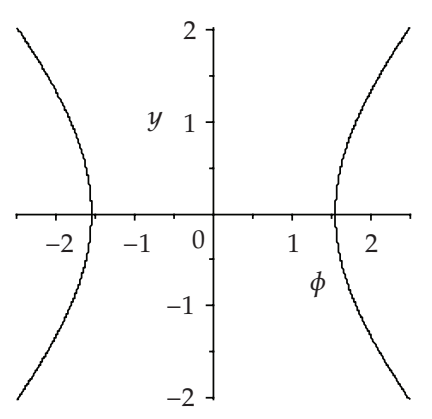

(a)

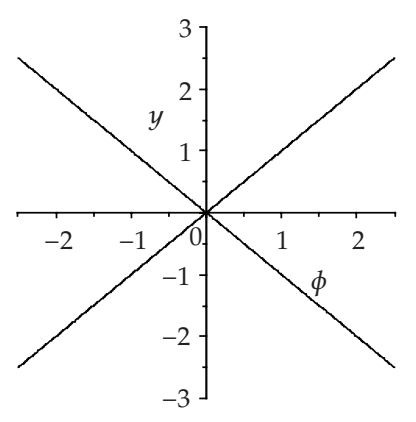

(b)

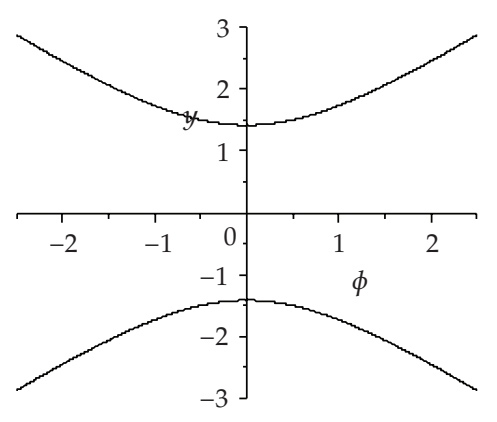

(c)

Figure 1: The phase portraits of system $(2.5)(c=\gamma)$. (a) $g<0$; (b) $g=0$; (c) $g>0$.

(3) if $g_{2}(c)<g<g_{1}(c)$, then system (2.5) has four equilibrium points $\left(\varphi_{0}^{-}, 0\right),\left(\varphi_{0}^{+}, 0\right)$, (c$\left.\gamma, y_{0}^{-}\right)$, and $\left(c-\gamma, y_{0}^{+}\right) .\left(c-\gamma, y_{0}^{-}\right)$and $\left(c-\gamma, y_{0}^{+}\right)$are two saddle points:

(i) if $c>\gamma$, then $\varphi_{0}^{-}<1 / 4(c-\gamma)<\varphi_{0}^{+} \leq c-\gamma \cdot\left(\varphi_{0}^{-}, 0\right)$ is a saddle point, while $\left(\varphi_{0}^{+}, 0\right)$ is a center point,

(ii) if $c<\gamma$, then $c-\gamma \leq \varphi_{0}^{-}<1 / 4(c-\gamma)<\varphi_{0}^{+}$. $\left(\varphi_{0}^{-}, 0\right)$ is a center point, while $\left(\varphi_{0}^{+}, 0\right)$ is a saddle point.

Specially, when $g=0$,

(i) if $c>\gamma$, then the three saddle points $\left(\varphi_{0}^{-}, 0\right),\left(c-\gamma, y_{0}^{-}\right)$, and $\left(c-\gamma, y_{0}^{+}\right)$form a triangular orbit which encloses the center point $\left(\varphi_{0}^{+}, 0\right)$,

(ii) if $c<\gamma$, then the three saddle points $\left(\varphi_{0}^{+}, 0\right),\left(c-\gamma, y_{0}^{-}\right)$, and $\left(c-\gamma, y_{0}^{+}\right)$form a triangular orbit which encloses the center point $\left(\varphi_{0}^{-}, 0\right)$;

(4) if $g=g_{1}(c)$, then system (2.5) has three equilibrium points $((c-\gamma) / 4,0),\left(c-\gamma, y_{0}^{-}\right)$, and $\left(c-\gamma, y_{0}^{+}\right) .((c-\gamma) / 4,0)$ is a degenerate center point, while $\left(c-\gamma, y_{0}^{-}\right)$and $\left(c-\gamma, y_{0}^{+}\right)$are two saddle points;

(5) if $g>g_{1}(c)$, then system (2.5) has two equilibrium points $\left(c-\gamma, y_{0}^{-}\right)$and $\left(c-\gamma, y_{0}^{+}\right)$, which are saddle points.

Corresponding to the case $c=\gamma$ and the case $c \neq \gamma$, we show the phase portraits of system (2.5) in Figures 1 and 2, respectively.

\section{Solitons, Peakons, and Periodic Cusp Wave Solutions}

Theorem 3.1. Given arbitrary constant $c \neq \gamma$, let $\xi=x-c t$, then

(1) when $0<g<g_{1}(c)$,

(i) if $c>r$, then (1.3) has the following smooth hump-like soliton solutions:

$$
\beta_{1}\left(\varphi_{1}^{+}\right)=\beta_{1}(\varphi) e^{-|\xi|} \text { for } \varphi_{0}^{-}<\varphi<\varphi_{1}^{+},
$$

(ii) if $c<\gamma$, then (1.3) has the following smooth valley-like soliton solutions:

$$
\beta_{2}(\varphi)=\beta_{2}\left(\varphi_{1}^{-}\right) e^{-|\xi|} \text { for } \varphi_{1}^{-}<\varphi<\varphi_{0}^{+},
$$




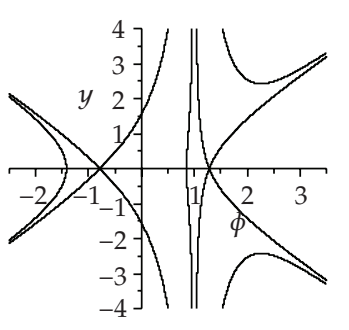

(a)

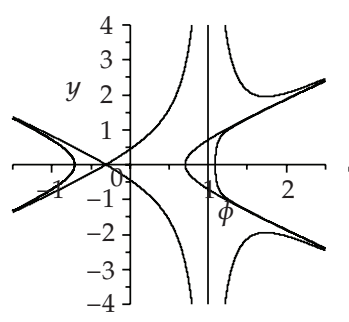

(e)

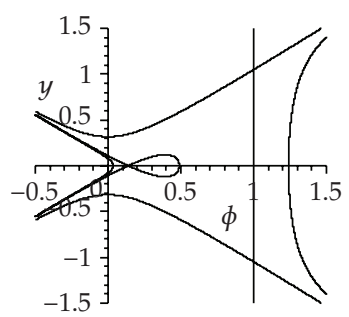

(i)

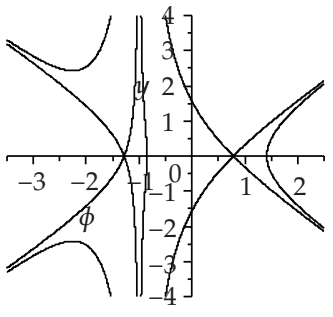

(b)

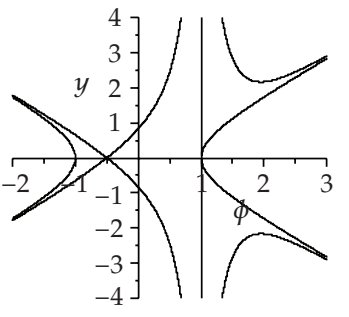

(c)

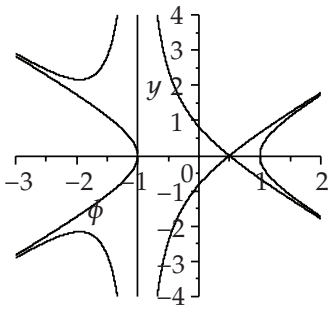

(d)

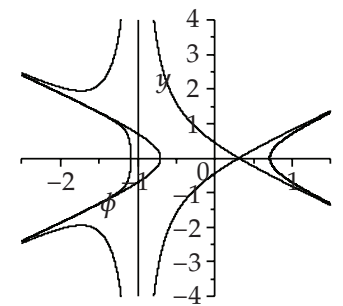

(f)

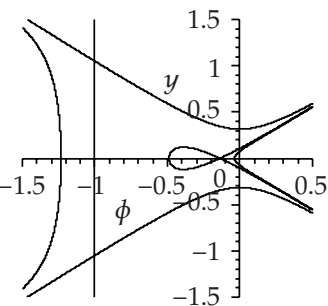

(j)

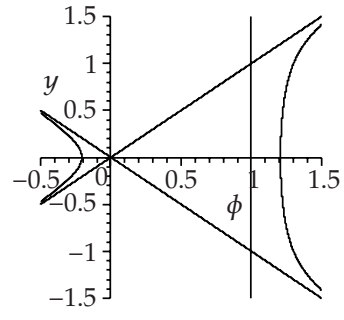

(g)

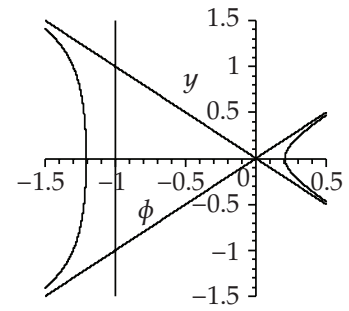

(h)

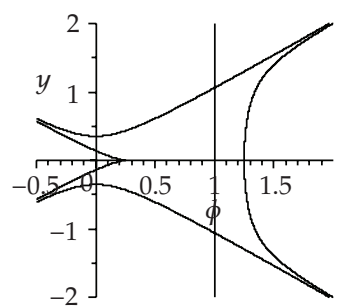

(k)

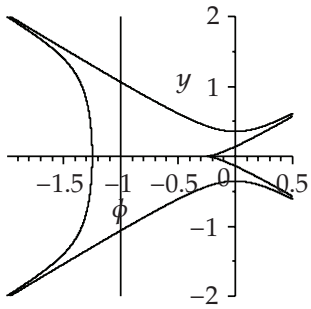

(1)

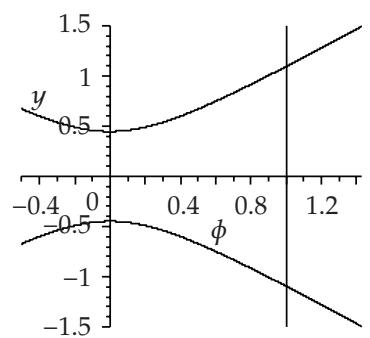

(m)

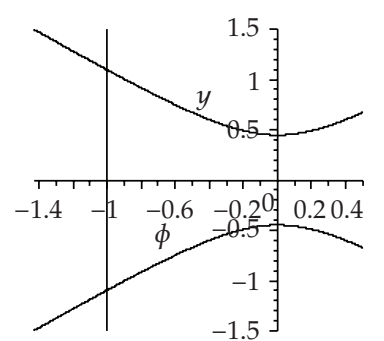

(n)

Figure 2: The phase portraits of system (2.5) $(c \neq \gamma)$. (a) $g<g_{2}(c), c>\gamma$; (b) $g<g_{2}(c), c<\gamma$; (c) $g=$ $g_{2}(c), c>\gamma$; (d) $g=g_{2}(c), c<\gamma$; (e) $g_{2}(c)<g<0, c>\gamma$; (f) $g_{2}(c)<g<0, c<\gamma$; (g) $g=0, c>\gamma$; (h) $g=0, c<\gamma$; (i) $0<g<g_{1}(c), c>\gamma$; (j) $0<g<g_{1}(c), c<\gamma$; (k) $g=g_{1}(c), c>\gamma$; $(1) g=g_{1}(c), c<\gamma$; (m) $g>g_{1}(c), c>\gamma ;(\mathrm{n}) g>g_{1}(c), c<\gamma$.

(2) when $g=0$, (1.3) has the following peaked soliton solutions:

$$
\varphi=(c-\gamma) e^{-|\xi|},
$$


(3) when $g_{2}(c)<g<0$,

(i) if $c>r$, then (1.3) has the following periodic cusp wave solutions:

$$
u(x, t)=\varphi_{3}(x-c t-2 n T) \quad \text { for }(2 n-1) T<x-c t<(2 n+1) T,
$$

(ii) if $c<\gamma$, then (1.3) has the following periodic cusp wave solutions:

$$
u(x, t)=\varphi_{4}(x-c t-2 n T) \quad \text { for }(2 n-1) T<x-c t<(2 n+1) T,
$$

where

$$
\begin{aligned}
\beta_{1}(\varphi) & =\frac{\left(2 \sqrt{\varphi^{2}+l_{1} \varphi+l_{2}}+2 \varphi+l_{1}\right)\left(\varphi-\varphi_{0}^{-}\right)^{\alpha_{1}}}{\left(2 \sqrt{a_{1}} \sqrt{\varphi^{2}+l_{1} \varphi+l_{2}}+b_{1} \varphi+l_{3}\right)^{\alpha_{1}}}, \\
\beta_{2}(\varphi) & =\frac{\left(2 \sqrt{\varphi^{2}+m_{1} \varphi+m_{2}}+2 \varphi+m_{1}\right)\left(\varphi-\varphi_{0}^{+}\right)^{\alpha_{2}}}{\left(2 \sqrt{a_{2}} \sqrt{\varphi^{2}+m_{1} \varphi+m_{2}}+b_{2} \varphi+m_{3}\right)^{\alpha_{2}}}, \\
l_{1} & =-\frac{3(c-\gamma)+\sqrt{(c-\gamma)^{2}-8 g}}{2} \\
l_{2} & =\frac{3(c-\gamma)^{2}-4 g+5(c-\gamma) \sqrt{(c-\gamma)^{2}-8 g}}{8} \\
l_{3} & =\frac{(c-\gamma)^{2}-4 g+3(c-\gamma) \sqrt{(c-\gamma)^{2}-8 g}}{2} \\
m_{1} & =-\frac{3(c-\gamma)-\sqrt{(c-\gamma)^{2}-8 g}}{2} \\
m_{2} & =\frac{3(c-\gamma)^{2}-4 g-5(c-\gamma) \sqrt{(c-\gamma)^{2}-8 g}}{8} \\
m_{3}= & \frac{(c-\gamma)^{2}-4 g-3(c-\gamma) \sqrt{(c-\gamma)^{2}-8 g}}{2} \\
& (c-\gamma)^{2}-8 g+3(c-\gamma) \sqrt{(c-\gamma)^{2}-8 g} \\
4 &
\end{aligned}
$$




$$
\begin{aligned}
a_{2} & =\frac{(c-\gamma)^{2}-8 g-3(c-\gamma) \sqrt{(c-\gamma)^{2}-8 g}}{4}, \\
b_{1} & =-(c-\gamma)-\sqrt{(c-\gamma)^{2}-8 g}, \\
b_{2} & =-(c-\gamma)+\sqrt{(c-\gamma)^{2}-8 g}, \\
\alpha_{1} & =-\frac{3(c-\gamma)+\sqrt{(c-\gamma)^{2}-8 g}}{2 \sqrt{(c-\gamma)^{2}-8 g+3(c-\gamma) \sqrt{(c-\gamma)^{2}-8 g}}}, \\
\alpha_{2} & =\frac{-3(c-\gamma)+\sqrt{(c-\gamma)^{2}-8 g}}{2 \sqrt{(c-\gamma)^{2}-8 g-3(c-\gamma) \sqrt{(c-\gamma)^{2}-8 g}}}, \\
\varphi_{3}(\xi) & =l_{+} e^{-|\xi|}+l_{-} e^{|\xi|} \quad \text { for } \sqrt{-g} \leq \varphi_{3} \leq c-\gamma, \\
\varphi_{4}(\xi) & =l_{+} e^{|\xi|}+l_{-} e^{-|\xi|} \quad \text { for } c-\gamma \leq \varphi_{4} \leq-\sqrt{-g}, \\
l_{ \pm} & =\frac{c-\gamma \pm \sqrt{(c-\gamma)^{2}+g}}{2}, \\
T & =\left|\ln (\sqrt{-g}+\sqrt{-2 g})-\ln \left(2 l_{-}\right)\right| \\
\varphi_{1}^{ \pm} & =\frac{3}{4}(c-\gamma) \pm \frac{1}{4} \sqrt{(c-\gamma)^{2}-8 g} \mp \frac{1}{2} \sqrt{(c-\gamma)^{2} \mp(c-\gamma) \sqrt{(c-\gamma)^{2}-8 g}} \\
&
\end{aligned}
$$

$\varphi_{0}^{+}$and $\varphi_{0}^{-}$are as in (2.9).

Before proving this theorem, we take a set of data and employ Maple to display the graphs of smooth solion, peaked soliton and periodic cuspon solutions of (1.3), see Figures 3, $4,5,6$ and 7 .

Proof. Usually, a solion solution of (1.3) corresponds to a homoclinic orbit of system (2.5), and a periodic travelling wave solution of (1.3) corresponds to a periodic orbit of system (2.5). The graphs of homoclinic orbit, periodic orbit of system (2.5), and their limit curves are shown in Figure 8.

(1) When $0<g<g_{1}(c), c>\gamma$, system (2.5) has a homoclinic orbit (see Figure $8(a)$ ). This homoclinic orbit can be expressed as

$$
y= \pm \frac{\left(\varphi-\varphi_{0}^{-}\right) \sqrt{\varphi^{2}+l_{1} \varphi+l_{2}}}{c-\gamma-\varphi} \text { for } \varphi_{0}^{-}<\varphi<\varphi_{1}^{+} .
$$

Substituting (3.7) into the first equation of system (2.3) and integrating along this homoclinic orbit, we obtain (3.1). 


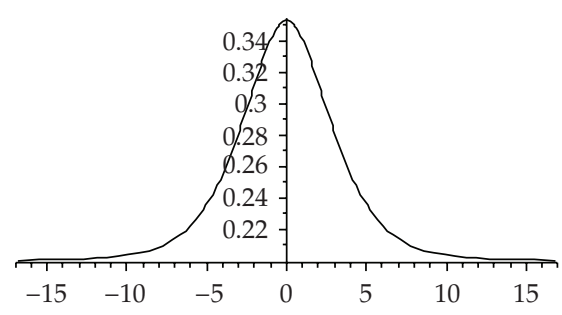

(a)

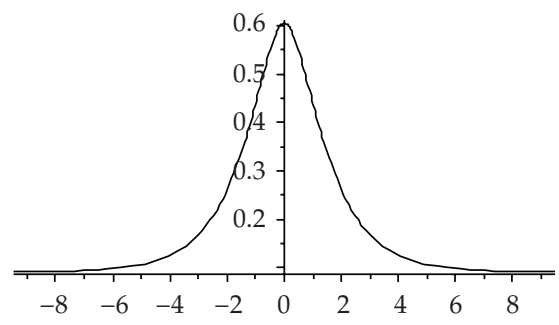

(c)

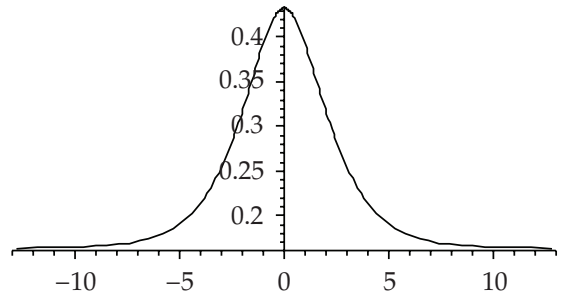

(b)

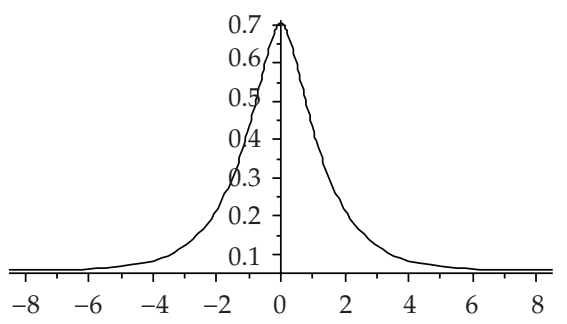

(d)

Figure 3: Smooth hump-like soliton solutions of $(1.3)(c=2, \gamma=1)$. (a) $g=0.12$; (b) $g=0.1$; (c) $g=0.075$; (d) $g=0.05$.

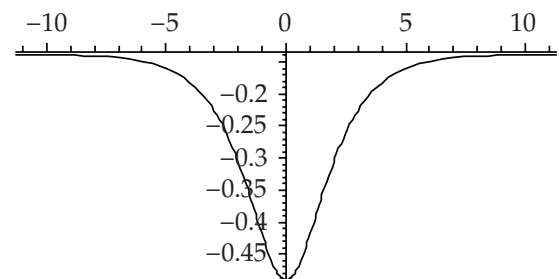

(a)

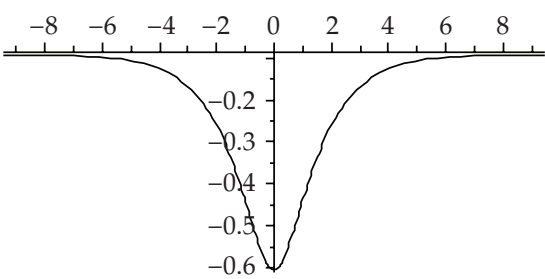

(c)

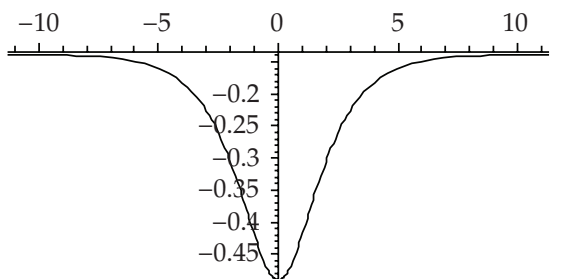

(b)

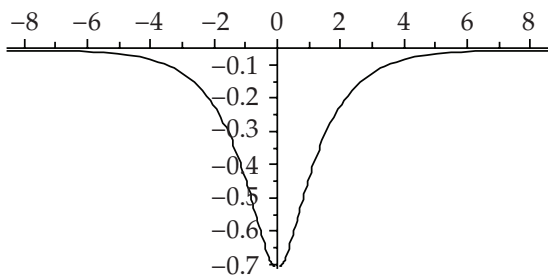

(d)

Figure 4: Smooth valley-like soliton solutions of (1.3) $(c=1, \gamma=2)$. (a) $g=0.12$; (b) $g=0.1$; (c) $g=0.075$; (d) $g=0.05$.

When $0<g<g_{1}(c), c<\gamma$, we can obtain (3.2) in similar way.

(2) When $g=0, c>\gamma$, system (2.5) has a homoclinic orbit that consists of the following three line segments (see Figure 8(c)):

$$
\begin{gathered}
y= \pm \varphi \text { for } \varphi_{0}^{-} \leq \varphi \leq \varphi_{1}^{+} \\
\varphi=c-\gamma \text { for }-\sqrt{(c-\gamma)^{2}+g} \leq y \leq \sqrt{(c-\gamma)^{2}+g} .
\end{gathered}
$$




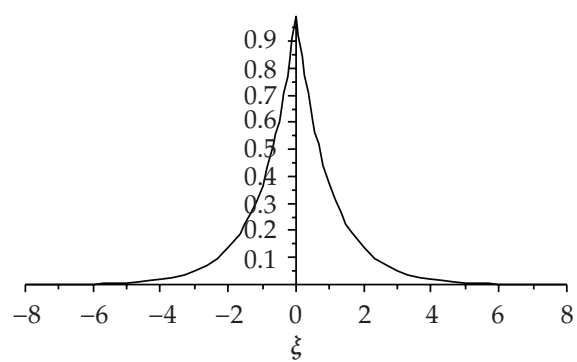

(a)

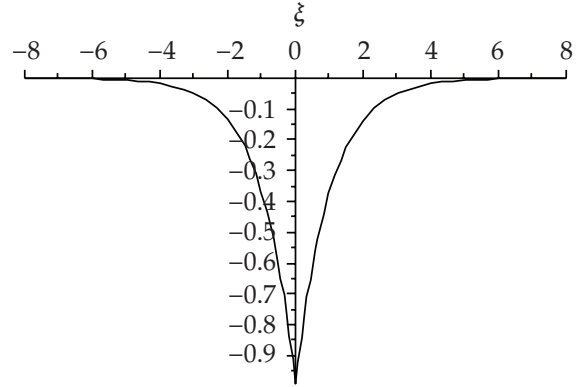

(b)

Figure 5: Peaked soliton solutions of (1.3). (a) $c=2, \gamma=1$; (b) $c=1, \gamma=2$.

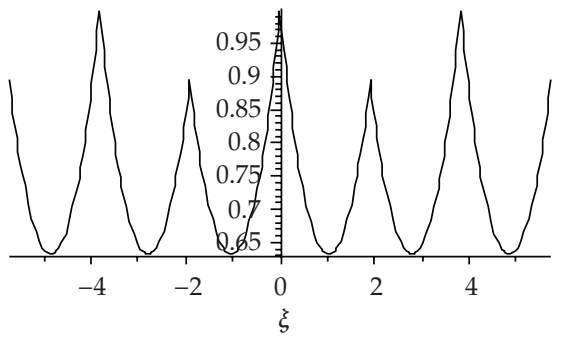

(a)

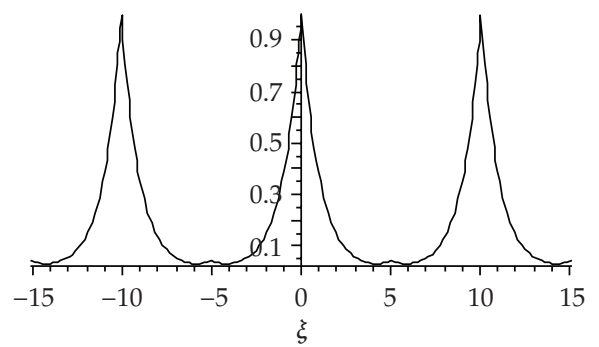

(c)

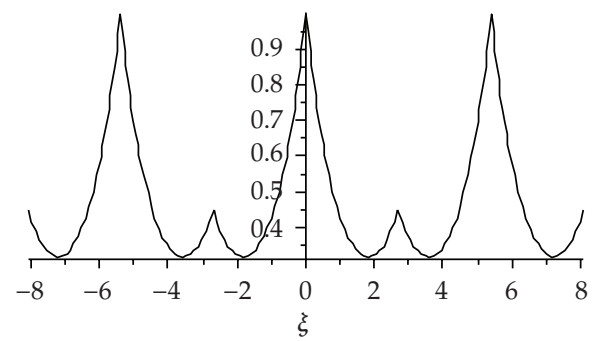

(b)

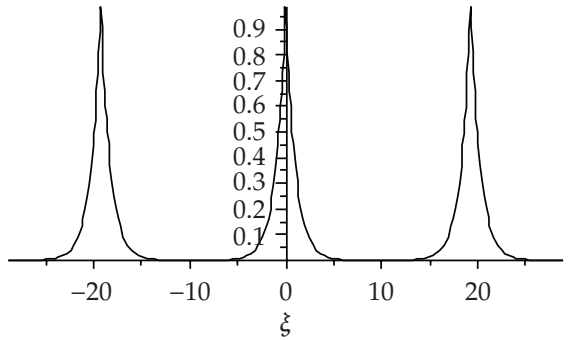

(d)

Figure 6: Period cuspon solutions of (1.3) $(c=2, \gamma=1)$. (a) $g=-0.4$; (b) $g=-0.1$; (c) $g=-0.01$; (d) $g=-0.0000001$.

Substituting (3.8) into the first equation of system (2.3) and integrating along this orbit, we obtain (3.3).

When $g=0, c<\gamma$, we can also obtain (3.3).

(3) When $g_{2}(c)<g<0, c>\gamma$, system (2.5) has a periodic orbit (see Figure 8(e)). This periodic orbit can be expressed as

$$
\begin{gathered}
y= \pm \sqrt{\varphi^{2}+g} \text { for } \sqrt{-g} \leq \varphi \leq c-\gamma \\
\varphi=c-\gamma \text { for }-\sqrt{(c-\gamma)^{2}+g} \leq y \leq \sqrt{(c-\gamma)^{2}+g} .
\end{gathered}
$$




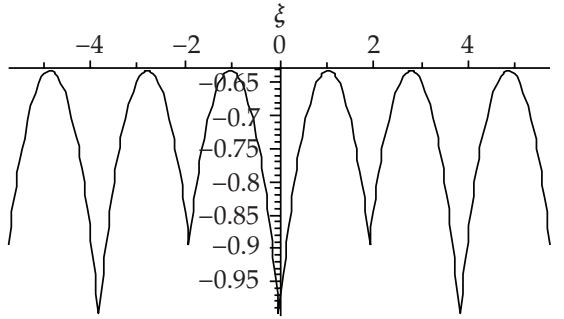

(a)

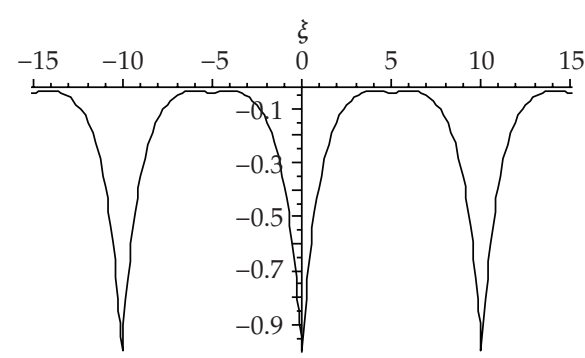

(c)

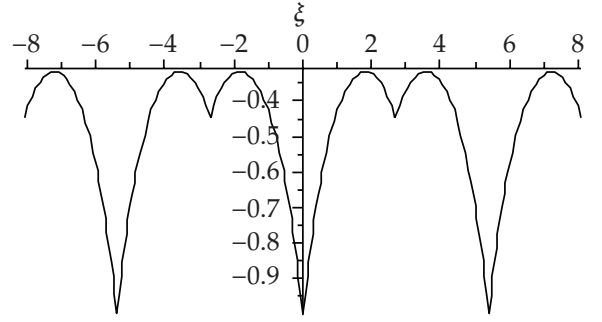

(b)

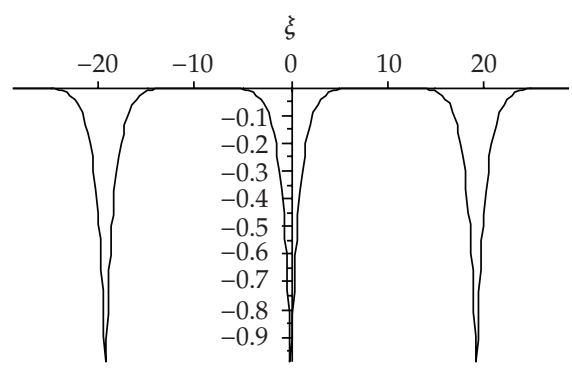

(d)

Figure 7: Period cuspon solutions of (1.3) $(c=1, \gamma=2)$. (a) $g=-0.4$; (b) $g=-0.1$; (c) $g=-0.01$; (d) $g=-0.0000001$.

Substituting (3.10) into the first equation of system (2.3) and integrating along this periodic orbit, we obtain (3.4).

When $g_{2}(c)<g<0, c<\gamma$, we can obtain (3.5).

Remark 3.2. From the above discussion, we can see that when $g<0, g \rightarrow 0$, the period of the periodic cusp wave solution becomes bigger and bigger, and the periodic cuspon solutions (3.4) and (3.5) tend to the peaked soliton solutions (3.3). When $g>0, g \rightarrow 0$, the smooth hump-like soliton solutions (3.1) and the smooth valley-like soliton solutions (3.2) lose their smoothness and tend to the peaked soliton solutions (3.3).

\section{Discussion}

In this paper, we obtain the solitons, peakons, and periodic cuspons of a generalized Degasperis-Procesi equation (1.3). These solitons denote the nonlinear localized waves on the shallow water's free surface that retain their individuality under interaction and eventually travel with their original shapes and speeds. The balance between the nonlinear steepening and dispersion effect under (1.3) gives rise to these solitons.

The peakon travels with speed equal to its peak amplitude. This solution is nonanalytic, having a jump in derivative at its peak. Peakons are true solitons that interact via elastic collisions under (1.3). We claim that the existence of a singular straight line for the planar dynamical system (2.3) is the original reason why the travelling waves lose their smoothness. each cusp.

Also, the periodic cuspon solution is nonanalytic, having a jump in derivative at its 


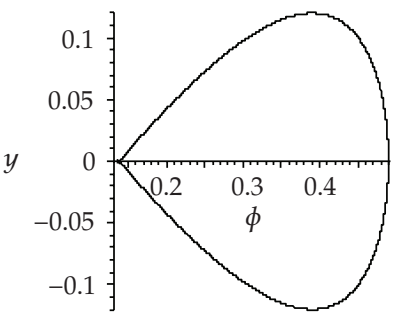

(a)

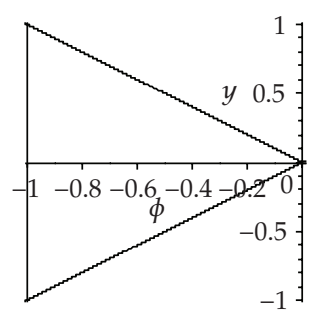

(d)

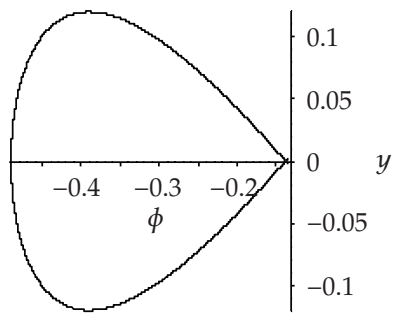

(b)

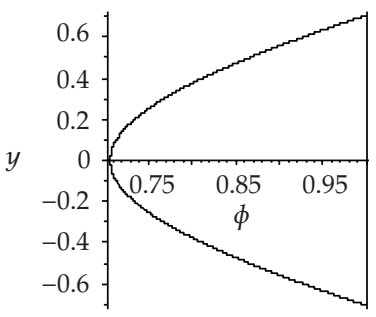

(e)

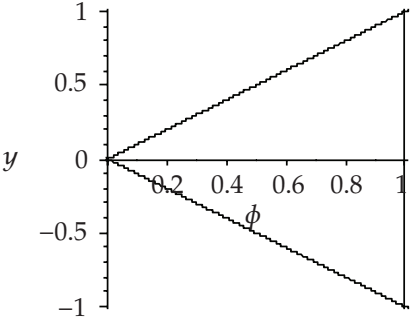

(c)

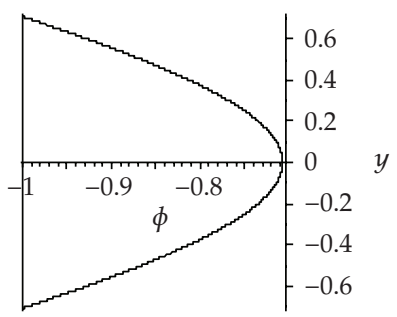

(f)

Figure 8: The orbits of system (2.5) connecting with the saddle points. (a) $0<g<g_{1}(c), c>\gamma$; (b) $0<g<$ $g_{1}(c), c<\gamma$; (c) $g=0, c>\gamma$; (d) $g=0, c<\gamma$; (e) $g_{2}(c)<g<0, c>\gamma$; (f) $g_{2}(c)<g<0, c<\gamma$.

\section{Acknowledgments}

The authors are deeply grateful to the anonymous referee for the careful reading of our manuscript and many constructive comments and valuable suggestions, which have helped them to improve it. This research was supported by NSF of China (No. 10771088) and Startup Fund for Advanced Talents of Jiangsu University (No. 09JDG013).

\section{References}

[1] A. Degasperis and M. Procesi, "Asymptotic integrability," in Symmetry and Perturbation Theory (Rome, 1998), A. Degasperis and G. Gaeta, Eds., pp. 23-37, World Scientific, River Edge, NJ, USA, 1999.

[2] A. Degasperis, D. D. Kholm, and A. N. I. Khon, "A new integrable equation with peakon solutions," Theoretical and Mathematical Physics, vol. 133, no. 2, pp. 1463-1474, 2002.

[3] Z. Yin, "On the Cauchy problem for an integrable equation with peakon solutions," Illinois Journal of Mathematics, vol. 47, no. 3, pp. 649-666, 2003.

[4] Z. Yin, "Global weak solutions for a new periodic integrable equation with peakon solutions," Journal of Functional Analysis, vol. 212, no. 1, pp. 182-194, 2004.

[5] Z. Yin, "Global solutions to a new integrable equation with peakons," Indiana University Mathematics Journal, vol. 53, no. 4, pp. 1189-1209, 2004.

[6] Z. Yin, "Global existence for a new periodic integrable equation," Journal of Mathematical Analysis and Applications, vol. 283, no. 1, pp. 129-139, 2003.

[7] Y. Zhou, "Blow-up phenomenon for the integrable Degasperis-Procesi equation," Physics Letters A, vol. 328, no. 2-3, pp. 157-162, 2004.

[8] J. Escher, Y. Liu, and Z. Yin, "Global weak solutions and blow-up structure for the Degasperis-Procesi equation," Journal of Functional Analysis, vol. 241, no. 2, pp. 457-485, 2006.

[9] Z. Yin, "Well-posedness, blowup, and global existence for an integrable shallow water equation," Discrete and Continuous Dynamical Systems. Series A, vol. 11, no. 2-3, pp. 393-411, 2004.

[10] Y. Liu and Z. Yin, "Global existence and blow-up phenomena for the Degasperis-Procesi equation," Communications in Mathematical Physics, vol. 267, no. 3, pp. 801-820, 2006. 
[11] V. O. Vakhnenko and E. J. Parkes, "Periodic and solitary-wave solutions of the Degasperis-Procesi equation," Chaos, Solitons \& Fractals, vol. 20, no. 5, pp. 1059-1073, 2004.

[12] Y. Matsuno, "Multisoliton solutions of the Degasperis-Procesi equation and their peakon limit," Inverse Problems, vol. 21, no. 5, pp. 1553-1570, 2005.

[13] Y. Matsuno, "Cusp and loop soliton solutions of short-wave models for the Camassa-Holm and Degasperis-Procesi equations," Physics Letters A, vol. 359, no. 5, pp. 451-457, 2006.

[14] H. Lundmark and J. Szmigielski, "Multi-peakon solutions of the Degasperis-Procesi equation," Inverse Problems, vol. 19, no. 6, pp. 1241-1245, 2003.

[15] J. Lenells, "Traveling wave solutions of the Degasperis-Procesi equation," Journal of Mathematical Analysis and Applications, vol. 306, no. 1, pp. 72-82, 2005.

[16] H. Lundmark, "Formation and dynamics of shock waves in the Degasperis-Procesi equation," Journal of Nonlinear Science, vol. 17, no. 3, pp. 169-198, 2007.

[17] J. Escher, Y. Liu, and Z. Y. Yin, "Shock waves and blow-up phenomena for the periodic DegasperisProcesi equation," Indiana University Mathematics Journal, vol. 56, no. 1, pp. 87-117, 2007.

[18] L. Yu, L. Tian, and X. Wang, "The bifurcation and peakon for Degasperis-Procesi equation," Chaos, Solitons \& Fractals, vol. 30, no. 4, pp. 956-966, 2006.

[19] D. Luo, X. Wang, D. Zhu, and M. Han, Bifurcation Theory and Methods of Dynamical Systems, vol. 15 of Advanced Series in Dynamical Systems, World Scientific, River Edge, NJ, USA, 1997. 


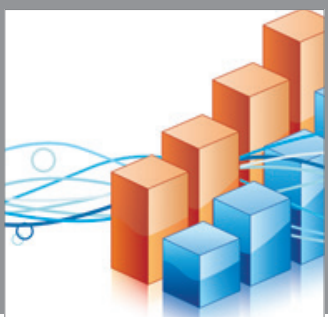

Advances in

Operations Research

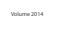

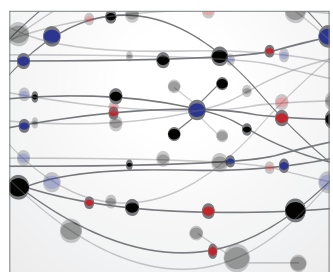

\section{The Scientific} World Journal
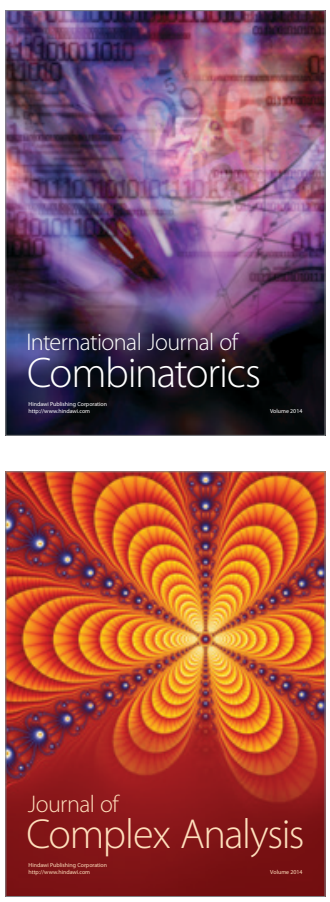

International Journal of

Mathematics and

Mathematical

Sciences
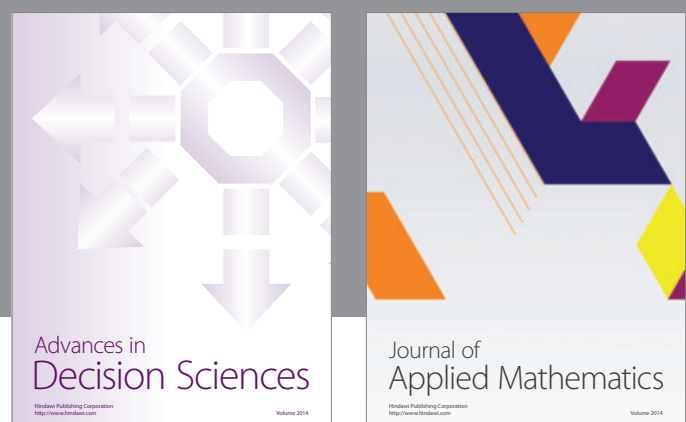

Journal of

Applied Mathematics
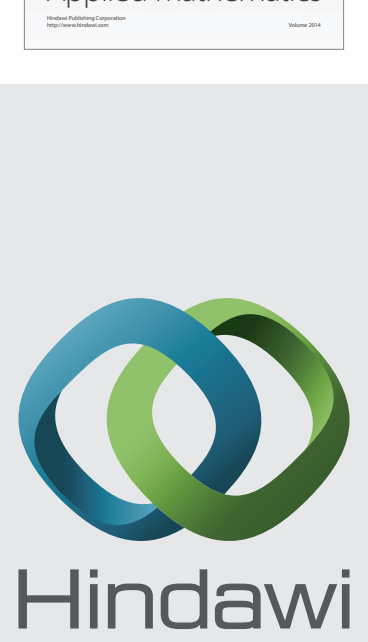

Submit your manuscripts at http://www.hindawi.com
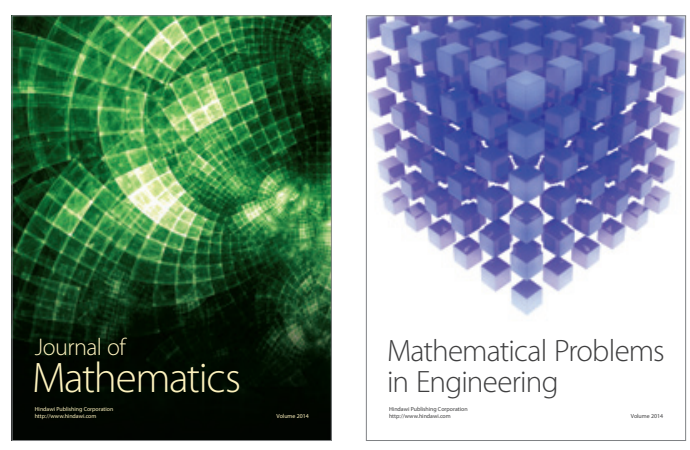

Mathematical Problems in Engineering
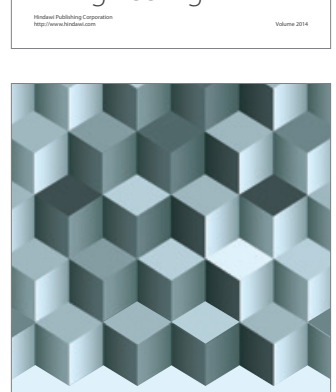

Journal of

Function Spaces
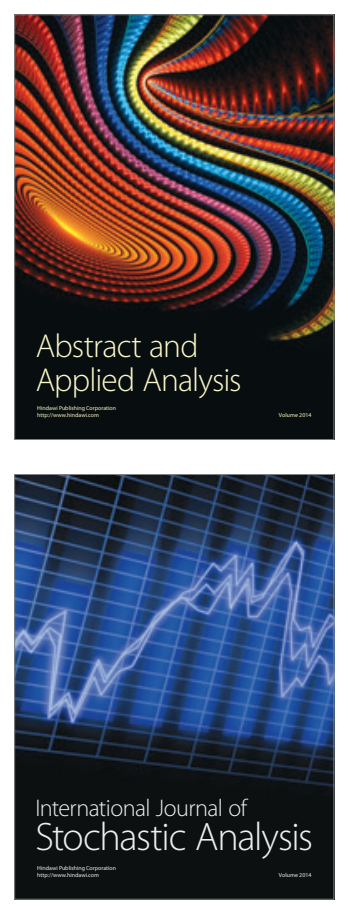

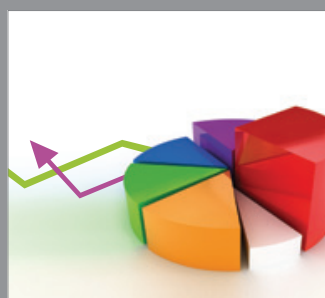

ournal of

Probability and Statistics

Promensencen
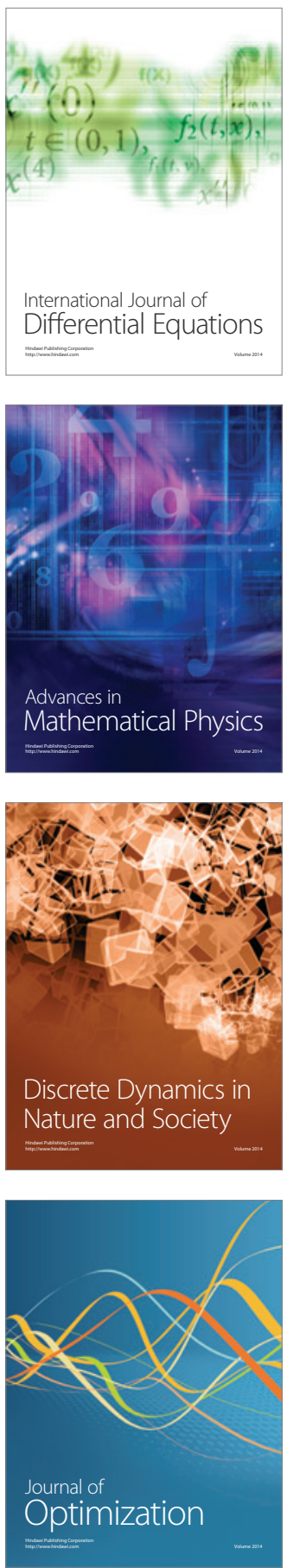